\title{
SHORT STORIES
}

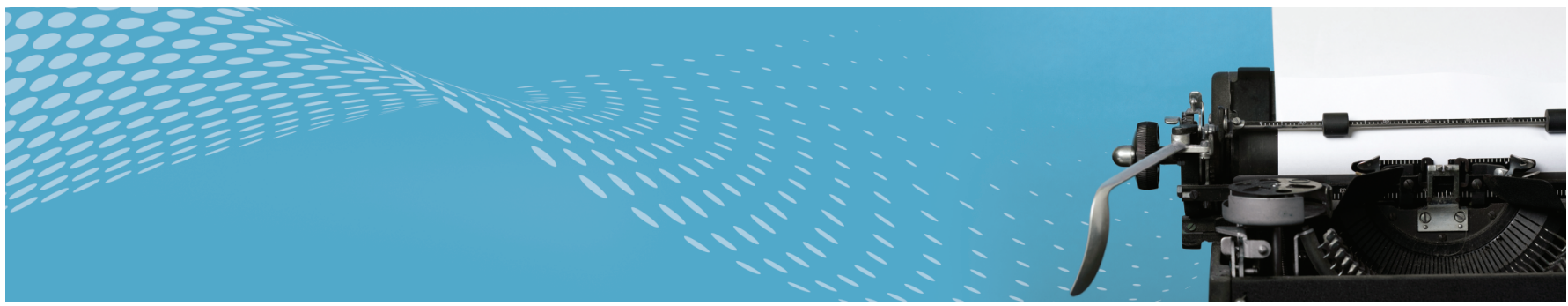

\section{Wild Wild Whitehead Danny Calegari}

Take a knot. Take a tube around the knot. Put a new knot in the tube, twisted around and clasping itself as in Figure 1 . The new knot goes once around the tube, and then "doubles back" and clasps itself. The new knot is the Whitehead double of the old.
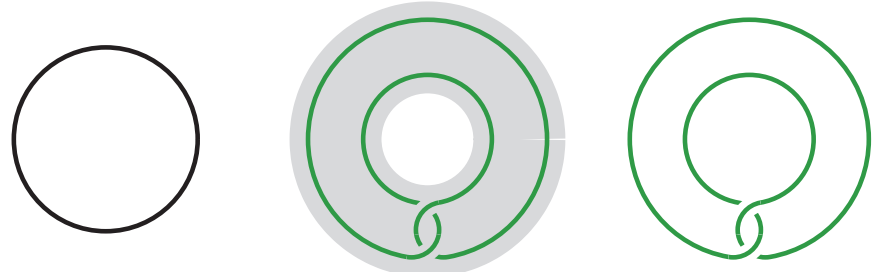

Figure 1. The green knot is the Whitehead double of the black knot.

Let's call the first knot $K_{0}$ and its Whitehead double $K_{1}$, and let's call the tube around $K_{0}$ (actually a solid torus) $N_{0}$. It makes sense to take the Whitehead double of any knot, but in Figure 1, $K_{0}$ is a trivial knot; i.e., it bounds an embedded disk in $S^{3}$. In this case, $K_{1}$ is trivial too: it bounds an embedded disk in $S^{3}$. But $K_{1}$ is knotted in $N_{0}$. Any embedded disk that $K_{1}$ bounds must go outside $N_{0}$.

On the other hand, $K_{1}$ is homotopically trivial in $N_{0}$; i.e., it bounds an immersed disk, one that crosses itself, but does not cross $N_{0}$. To see this, just push one of the clasps of $K_{1}$ through the other one; this undoes the knotting, and the

Danny Calegari is a professor of mathematics at the University of Chicago. His email address is dannyc@math . uchicago.edu.

For permission to reprint this article, please contact: reprint-permission@ams.org.

DOI: https://doi.org/10.1090/noti1837 result can be shrunk down to a point. The track of the knot $K_{1}$ during this process sweeps out an immersed disk.

In terms of the fundamental group, a knot $K$ in a space $X$ determines a conjugacy class $[K]$ in the fundamental group $\pi_{1}(X)$. Now, $\pi_{1}\left(N_{0}\right)=\mathbb{Z}$, and since $K_{1}$ bounds an immersed disk, $\left[K_{1}\right]$ is trivial in $\pi_{1}\left(N_{0}\right)$.

We can keep going. Let $N_{1}$ be a tube around $K_{1}$, thin enough to fit in $N_{0}$, and let $K_{2} \subset N_{1}$ be the Whitehead double of $K_{1}$. And so on. Each $K_{i}$ bounds an embedded disk in $S^{3}$, but each of these disks must go (many times!) all the way outside $N_{0}$.

The tubes get thinner and thinner as we go, and longer and longer. Consequently, the knots must get longer and longer too: Each $K_{n}$ must wind back and forth at least $2^{n}$ times around $N_{0}$, clasping itself in a complicated way at the end. The infinite intersection $\bigcap_{i} N_{i}$ is called the Whitehead continuum, which we write $W h$, see Figure 2 . The Whitehead continuum is connected but not path connected. It has an entangled dyadic Cantor set of "strands" that wind around $N_{0}$.

The complement $S^{3}-W h$ is an open 3-manifold called the Whitehead manifold. It turns out that $S^{3}-W h$ is contractible but not homeomorphic to a 3-ball. Let's see why.

The outside of $N_{0}$ in $S^{3}$ is another solid torus $N_{0}^{\prime}$, whose core is a knot $K_{0}^{\prime}$ linking $K_{0}$ in a Hopf link. [ $\left.K_{0}^{\prime}\right]$ is the generator of $\pi_{1}\left(S^{3}-N_{0}\right)=\pi_{1}\left(N_{0}^{\prime}\right)=\mathbb{Z}$. The knots $K_{0}^{\prime}$ and $K_{1}$ together form a 2-component link called the Whitehead link. This link is symmetric: we can isotope it around and interchange $K_{0}^{\prime}$ and $K_{1}$; see Figure 3 .

Since $\left[K_{1}\right]$ is trivial in $\pi_{1}\left(N_{0}\right)=\pi_{1}\left(S^{3}-N_{0}^{\prime}\right)$, it follows by symmetry that $\left[K_{0}^{\prime}\right]$ is trivial in $\pi_{1}\left(S^{3}-N_{1}\right)$. Consequently the inclusion map $S^{3}-N_{0} \rightarrow S^{3}-N_{1}$ 

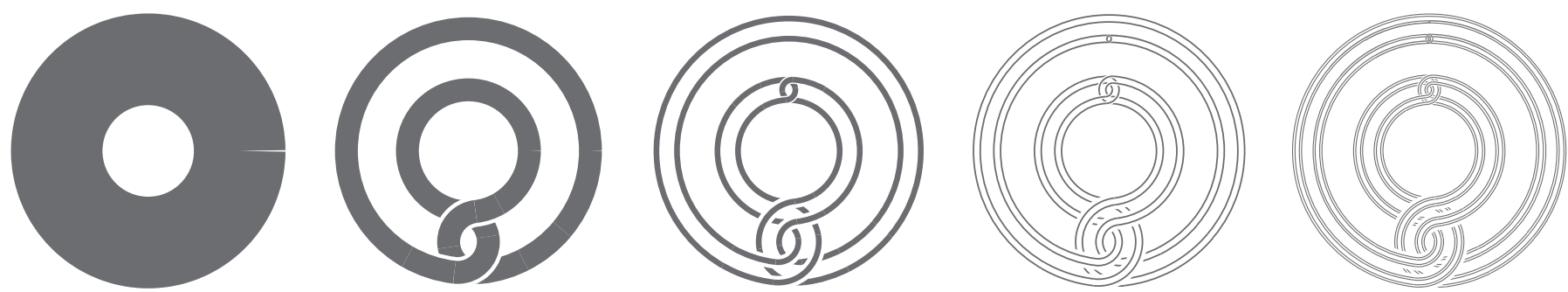

Figure 2. Successive approximations converge to Wh.
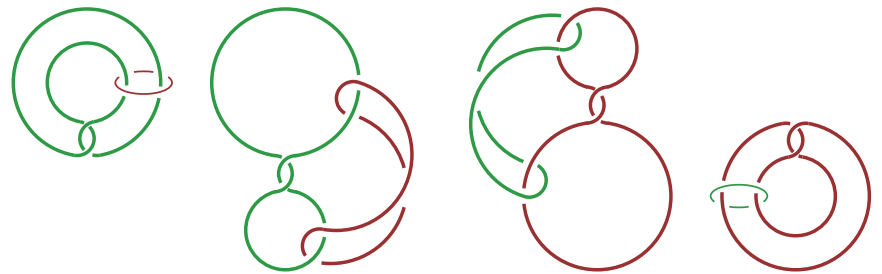

Figure 3. The Whitehead link is symmetric.

induces the zero map on $\pi_{1}$. Each $N_{i}$ is unknotted in $S^{3}$, and each $N_{i+1}$ sits in $N_{i}$ the same way that $N_{1}$ sits in $N_{0}$. So each $\pi_{1}\left(S^{3}-N_{i}\right)=\mathbb{Z}$, and each inclusion $S^{3}-N_{n} \rightarrow S^{3}-N_{n+1}$ induces the zero map on $\pi_{1}$. Taking a direct limit, $\pi_{1}\left(S^{3}-W h\right)$ itself is trivial. A similar argument shows that all the homotopy groups of $S^{3}-W h$ vanish, and it is contractible.

On the other hand, the complement $S^{3}-W h-N_{0}^{\prime}=$ $N_{0}-W h$ has an infinitely generated fundamental group; each $\pi_{1}\left(N_{i}-N_{i+1}\right)$ is complicated (it contains free groups of every rank), and all of them include as subgroups of $\pi_{1}\left(N_{0}-W h\right)$. This shows that $S^{3}-W h$ is not a ball.

Now let's compactify $S^{3}-W h$ by adding a single point at infinity. This compact space can also be thought of as the quotient space $S^{3} / W h$ that we get by crushing Wh to a single point. Because $S^{3}-W h$ is not a ball, $S^{3} / W h$ is not a manifold. However-remarkably-it is a manifold factor: the product $\left(S^{3} / W h\right) \times \mathbb{R}$ is homeomorphic to $S^{3} \times \mathbb{R}$ !

How can this possibly be???

First, each $N_{1}$ slice can be unknotted by a tiny perturbation in $N_{0} \times \mathbb{R}$. To distinguish the $\mathbb{R}$ factor and, for the sake of brevity, we refer to it as the "time" coordinate (this is purely a notational convenience). In this language, we unclasp $N_{1}$ from itself by nudging one clasp very slightly forward into the future, and the other very slightly back into the past. After the nudge, $N_{1}$ will not clasp itself, but it will clasp a "future" $N_{1}$ on one side, and a "past" $N_{1}$ on the other. Instead of $N_{1}$ clasping itself in a circle, we get a chain of successive $N_{1}$ 's, each clasping the next, in a slowly ascending spiral. Let's let $\epsilon / 4$ be the size of the perturbations of each clasp in the time direction, so that the projection of each $N_{1}$ to the time coordinate after it's been nudged has total length $\epsilon / 2$.
Nudging adjusts points in $N_{1} \times \mathbb{R}$ by sliding each point $\times \mathbb{R}$ slightly backward or forward in time. Nudging extends to a self-homeomorphism $v$ of $N_{0} \times \mathbb{R}$, fixed on the boundary.
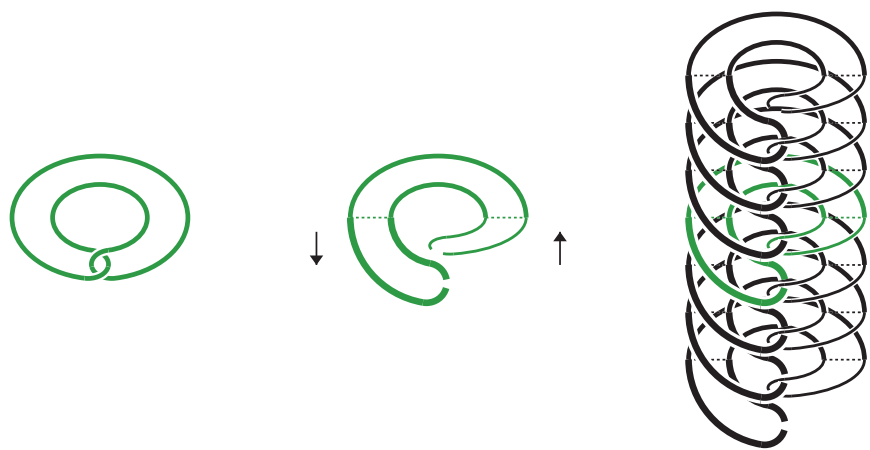

Figure 4. Folding the clasps of each $N_{1}$ back and forth in time nudges the union of all $N_{1}$ 's into a collection of spirals.

By the way, there's not just one spiral-there's a circle's worth of them, filling the whole of $N_{1} \times \mathbb{R}$. Two slices $v\left(N_{1} \times t\right), v\left(N_{1} \times s\right)$ are in the same spiral if and only if $t-s$ is an integer multiple of $\epsilon / 2$.

After nudging, the next move will straighten out this and every other spiral so that its projection to the $S^{3}$ factor is small (let's say for concreteness it has diameter $<\epsilon / 2$ ) without affecting the projection to the $\mathbb{R}$ factor.

The cylinder $K_{0} \times \mathbb{R} \subset S^{3} \times \mathbb{R}$ has polar coordinates $(\theta, t)$ where $\theta \in \mathbb{R} / \mathbb{Z}$. Extend these polar coordinates to a small tubular neighborhood of $K_{0} \times \mathbb{R}$ containing $N_{1} \times \mathbb{R}$, with closure contained in the interior of $N_{0} \times \mathbb{R}$.

We can "untwist" every spiral simultaneously by the map

$$
(\theta, t) \rightarrow(\theta-2 t / \epsilon, t)
$$

on our small tubular neighborhood. Twisting extends to a self-homeomorphism $T$ of $N_{0} \times \mathbb{R}$, once again fixed on the boundary.

In summary, first we nudge, then we twist. After doing this, every $\tau \mathcal{V}\left(N_{1}\right)$ slice projects to subsets of diameter at most $\epsilon / 2$ in both the $\mathbb{R}$ and the $S^{3}$ directions. So $\tau \mathcal{V}\left(N_{1}\right)$ has diameter at most $\epsilon$.

In other words, $h_{1}:=\tau \nu$ simultaneously shrinks all the $N_{1}$ slices in $S^{3} \times \mathbb{R}$ as small as we like, while keeping $\left(S^{3}-N_{0}\right) \times \mathbb{R}$ fixed pointwise. 

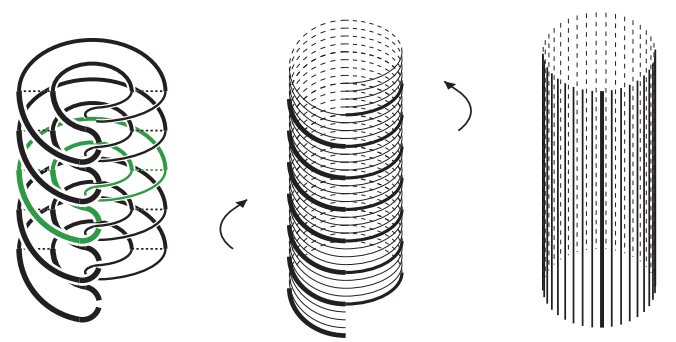

Figure 5. Screw the top and bottom in opposite directions like you're taking the lid off a pickle jar.

Take a sequence $\epsilon_{i} \rightarrow 0$, and repeat this operation for each $i>1$ in place of 1 with $\epsilon_{i}$ in place of $\epsilon$. We get a sequence of self-homeomorphisms $h_{i}: S^{3} \times \mathbb{R} \rightarrow S^{3} \times$ $\mathbb{R}$, each supported in $N_{i-1} \times \mathbb{R}$, as a composition of a nudge-and-twist $h_{i}:=\tau_{i} v_{i}$. Each $N_{i}$ slice gets smaller and smaller in diameter as we apply consecutive $h_{i}$ 's. Under application of successive $h_{i}$ 's, the orbit of every point is a Cauchy sequence, and the infinite composition

$$
h:=\lim _{i \rightarrow \infty} h_{i} h_{i-1} \cdots h_{1}: S^{3} \times \mathbb{R} \rightarrow S^{3} \times \mathbb{R}
$$

is well-defined and continuous.

For any compact subset $X$ of $S^{3}-W h$ the restriction of $h$ to $X$ is the composition of finitely many homeomorphisms, so the restriction of $h$ to $\left(S^{3}-W h\right) \times \mathbb{R}$ is a homeomorphism. On the other hand, each Wh slice is successively shrunk smaller and smaller by successive $h_{i}$, so in the end $h$ crushes each Wh slice to a point, and $h$ factors as $h=g \pi$,

$$
S^{3} \times \mathbb{R} \stackrel{\pi}{\rightarrow}\left(S^{3} / W h\right) \times \mathbb{R} \stackrel{g}{\rightarrow} S^{3} \times \mathbb{R}
$$

where $\pi: S^{3} \times \mathbb{R} \rightarrow\left(S^{3} / W h\right) \times \mathbb{R}$ is the quotient map, and $g$ is the homeomorphism we've been looking for.

AUTHOR'S NOTE. The main theorem in this article and its proof are both well-known, and not due to me! They are due to J. Andrews and L. Rubin, Bull. Amer. Math. Soc. 71(1965), 675-677.

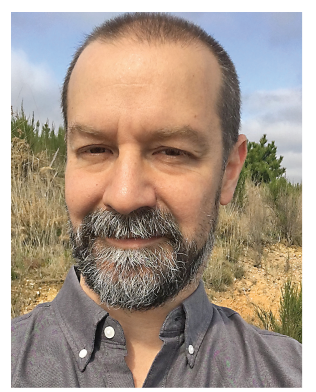

Danny Calegari

\section{Credits}

All images are courtesy of Danny Calegari.

\section{GET MORE FROM YOUR JOURNALS}

\section{Learn about AMS MathViewer:}

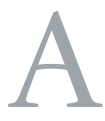
$\mathrm{n}$ interactive, dual-panel reading experience for journals published by the American Mathematical Society.

AMS MathViewer's responsive HTML format perfectly renders mathematical content on desktop, tablet, or phone. Click on embedded links to formulas, theorems, figures, and references to view them in the second panel, in tandem with the original text.

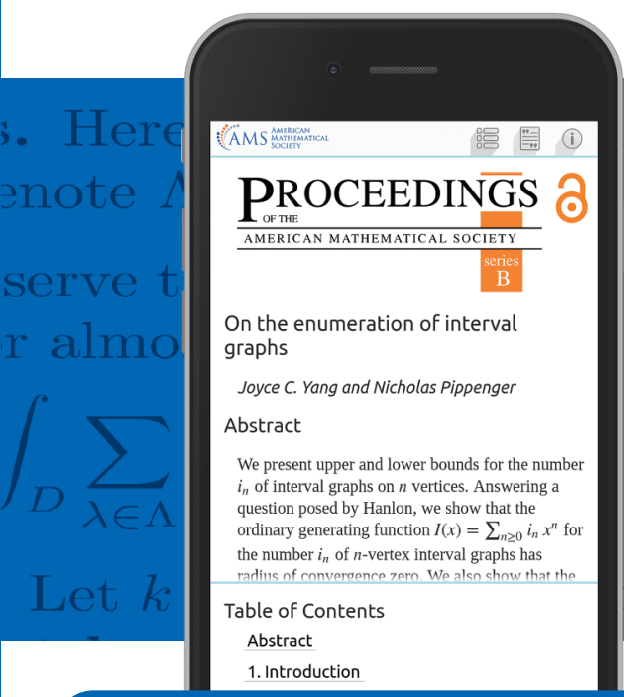

CURRENTLY AVAILABLE FOR:

Transactions of the AMS - Series B

Proceedings of the AMS - Series B

Journal of the American Mathematical Society

Mathematics of Computation

(Starting with 2019 volumes and

select articles from past issues.)

Keep an eye out for additional journals that will become available throughout the year.

www.ams.org/AMSMathViewer

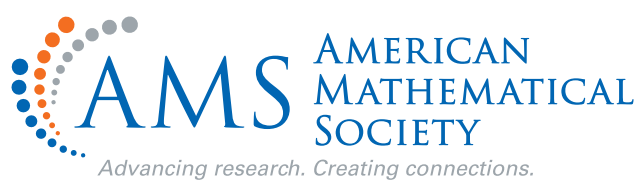

